\title{
Supernovae, Rotation, and Bipolar Explosions
}

\author{
A. Burrows ${ }^{\mathrm{a} *}$, R. Walder ${ }^{\mathrm{b}}$, C.D. Ott ${ }^{\mathrm{c}}$, and E. Livne ${ }^{\mathrm{d}}$ \\ aDepartment of Astronomy, The University of Arizona, Tucson, AZ 85721; \\ aburrows@as.arizona.edu \\ ${ }^{\mathrm{b}}$ Department of Astronomy, The University of Arizona, Tucson, AZ 85721; \\ rwalder@as.arizona.edu \\ ${ }^{c}$ Max-Planck-Institut für Gravitationsphysik, \\ Albert-Einstein-Institut, Golm/Potsdam, Germany; cott@aei.mpg.de \\ ${ }^{\mathrm{d}}$ Racah Institute of Physics, The Hebrew University, Jerusalem, Israel; \\ eli@frodo.fiz.huji.ac.il
}

In this paper, we discuss the possible role of rotation in supernova blast energetics and morphology, and speculate on the origin of Cas A's and SN1987A's ejecta fields. Two "explosive" phenomena may be associated with most core collapses, the neutrinodriven supernova itself and an underenergetic jet-like ejection that follows. The latter may be a magnetic wind that easily penetrates the debris created by the much more energetic supernova. We speculate that many core-collapse supernova remnants should have sub-dominant jet-like features. In Cas A, we associate this sub-dominant collimated wind with the "jet/counter-jet" structure observed. We suggest that the actual Cas A explosion itself is at nearly right angles to this jet, along a rotation axis that coincides with the bulk of the ejecta, the iron lobes, and the putative direction of motion of the point source. It may be that when rotation becomes sufficiently rapid that the strong-neutrinodriven-supernova/weak-jet duality switches to a strong-MHD-jet scenario that might be associated with hypernovae, and in some cases GRBs. Finally, we present a calculation using a new 2D multi-group, flux-limited radiation/hydrodynamics code we have recently developed for the simulation of core-collapse supernovae. We discuss the rotation-induced anisotropy in the neutrino radiation field, neutrino heating, and the neutrino flux vectors and speculate on rotation's possible role in the supernova mechanism.

\footnotetext{
*We acknowledge discussions with Todd Thompson, Jeremiah Murphy, Casey Meakin, Itamar Lichtenstadt, and Moath Jarrah. Support for this work is provided in part by the Scientific Discovery through Advanced Computing (SciDAC) program of the DOE, grant number DE-FC02-01ER41184. In addition, we thank Jeff Fookson and Neal Lauver of the Steward Computer Support Group for their invaluable help with the local Beowulf cluster and acknowledge the use of the NERSC/LBNL/seaborg and ORNL/CCS/cheetah machines.
} 


\section{Neutrino-Driven Mechanism Preferred Over MHD Jet-Driven Mechanism}

It has been suggested that the association between gamma-ray bursts (GRBs), which seem to be powered by relativistic jets, and the Type Ic subclass of supernovae, as well as the existence of hypernovae [1], means that the generic supernova is powered my MHD jets $[2,3]$. However, there are many reasons to doubt this conclusion. The GRB rate in the universe is close to one or two per day, but the universe's supernova rate from the death of stars more massive than $\sim 8 \mathrm{M}_{\odot}$ is close to one per second. With a beaming angle of $\sim 5^{\circ}$ $(1 / 500$ th of the sky), the GRB/SNe ratio is only $\sim 0.5 \%$. This is significantly lower than the fraction of all supernovae that are Type Ic's. In addition, the spectra of canonical Type Ic supernovae do not show the high velocities and high ${ }^{56} \mathrm{Ni}$ masses associated with the bumps in GRB afterglows and SN1998bw-like explosions [1,4]. This implies that the supernovae paired with GRBs are special [5]. Hence, though evidence is accumulating that GRBs are connected with the deaths of a subset of massive stars, that subset is likely to be small. For instance, a major contender for the GRB engine, the collapsar model [6], could involve only those stars more massive than $80 \mathrm{M}_{\odot}$ that also have "rapidly" rotating cores at collapse. The massive star mass function places $\sim 1-2 \%$ of all massive stars above this cutoff, still enough to explain GRBs even if only a minority of them are fast rotators. Moreover, it has been suggested that the only major difference between supernovae and GRBs is the presence or absence of hydrogen and helium envelopes. But, as already mentioned, the typical Type Ib/c supernova light curves, line profiles, spectra, and ejection velocities look very different from those inferred by analyzing hypernovae and GRB afterglows.

It has been claimed that the large polarizations seen in many Type Ic supernovae [7] are evidence that the necessary asymmetry is due to a jet morphology. However, any explosion asymmetry, such as has been a feature of supernova theory for the last decade [8-12], could explain the magnitude of the measured polarizations. The instabilities that are generic to neutrino-driven models produce large-scale fingering and asphericities with 1:2 or 1:3 axis ratios [8]. However, the angle of polarization observed in SN1987A is roughly correlated with the axes of its encircling rings and these rings are likely associated with a rotation axis. Therefore, it is reasonable to conclude that SN1987A's explosion asymmetry, as inferred from polarization studies, is correlated with the rotation of the core. In addition, SN1987A's debris morphology is bipolar, and is along the same axis [13]. However, this does not mean that supernovae are driven by jets. In fact, introducing rotation into the standard neutrino-driven paradigm of core-collapse supernovae can produce quasi-bipolar explosions of the requisite character $(\S 4 ;[14,15])$. The bipolarity is not strongly collimated $\left(30^{\circ}-60^{\circ}\right)$, unless the rotation rate is large [15].

With rapid rotation, MHD power could be dynamically influential, but this may not be the normal situation. A picture we favor is that most supernovae are neutrino-driven, with the aspherical blast morphology influenced by slow to modest rotation. This will give a bipolar structure to the explosion debris and polarize the inner ejecta. It will also result in a bipolar distribution of the iron-group elements. Such a morphology and element distribution are seen in Cas A $(\S 2 ;[16])$. In the neutrino-driven scenario, not only does rotation impose modest bipolarity, but it enhances the chances for explosion and increases the explosion energy $(\S 4)$. Moreover, the neutrino-driven supernova may be 
accompanied by a sub-dominant MHD jet with a low luminosity and total energy, that may nevertheless emerge from the core to modify the debris. This jet may simply be the expected protoneutron star wind that follows the supernova [8], modified by rotation and magnetic hoop stresses. Hence, there may be two "explosions" at different times: one, the neutrino-driven supernova and, the other (a weaker one), the MHD jet or B-field-modified protoneutron star wind. For most supernovae, the latter two would be of minor dynamical import.

However, when the rotation rate is very fast, MHD processes could take over, particularly if a massive core in a massive progenitor inhibits the neutrino-driven mechanism. In that case, a hypernova and/or a GRB could result (if most of the outer envelope has already been lost; perhaps also if a black hole first forms), driven by a narrow $\left(\sim 3^{\circ}-10^{\circ}\right)$ MHD jet of high power. Rotation naturally amplifies B-fields, either by the MRI $[3,17]$, flux wrapping, or more conventional dynamo action, and there will be a critical rotation rate and degree of differential rotation above which MHD power and power densities will take over from those of neutrino processes. But such high spin rates may not be common, making hypernovae and GRBs rare. In addition, for suitably massive progenitors with slowly rotating cores, the supernova may abort, a black hole may form, and a hypernova/GRB may not follow. This may be the normal evolutionary path for most very massive stars $\left(>40 \mathrm{M}_{\odot}\right.$ ??).

\section{The Cas A Blast Morphology: Clues to the Supernova Engine}

Cas A shows a jet in its northeast, and a "counter-jet" in its southwest [16] that some have suggested is a signature of a jet-driven explosion. However, most of the blast mass and energy is actually nearly perpendicular to this jet, concentrated near the north and south poles $[18,19]$. Furthermore, iron that must be associated with the central engine is distributed in two caps in the same locations $[20,16]$. We surmise that the axis connecting these iron-rich, mass-rich, and energy-rich regions is the real axis of the supernova and that it exploded in quasi-bipolar fashion roughly along a rotation axis connecting the northnorthwest/south-southeast directions. The neutrino mechanism with some rotation can explain this morphology and debris pattern $(\S 4 ; \S 5)$. The jet/counter-jet structures would not have driven the supernova, would be underenergetic, and would have emerged after the supernova explosion into the prepared supernova debris. However, it would be natural for the jet axis to be a rotation axis, and since in Cas A this axis is roughly perpendicular to the axis we are identifying with the bipolar explosion, the rotation axis of the engine would have to have precessed between the explosion and the emergence of the "MHD" jet or collimated protoneutron star wind. This is problematic, but, nevertheless, the rest of the story we outline for Cas A retains its appeal. Alternately, the jet/counter-jet axis could indicate the direction of the magnetic dipole; a finite angle between the rotation axis and the magnetic dipole axis is a central feature of pulsar theory. If this were the case, there would be added constraints on the ratio of the jet duration and the rotation period. Note that the inferred direction of motion (kick?) of the central X-ray point source in Cas A [21] is along the axis we identify as the axis of explosion, not the "jet/counter-jet" axis. This point source is also inferred to be moving in the "blue-shifted" direction, mildly towards us. In this scenario, the kick would be due to hydrodynamic recoil and (perhaps) 
gravitational attraction with the inner debris [12] in the context of a mildly top-bottom asymmetric $[22,12]$ bipolar explosion. Encouragingly, estimates are that the "red-shifted" cap of mass and iron in the north/northwest has a bit more mass and kinetic energy than the "blue-shifted" cap, consistent with the recoil interpretation $[18,19]$. The gravitational impulses might be accompanied (in 3D) by gravitational torques that could precess the rotation axis of the settling nascent protoneutron star.

\section{The Neutrino-Driven Explosion Mechanism}

The prompt bounce never leads to direct explosion in $1 \mathrm{D}, 2 \mathrm{D}$, or $3 \mathrm{D}$; neutrino losses and photodissociation by the shock debilitate it, even for the lowest mass progenitors and accretion-induced collapse. In the Chandrasekhar context, there is just too much mass between the place the shock originates $\left(\sim 0.6 \mathrm{M}_{\odot}\right)$ and the outer boundary $\left(\geq 1.2 \mathrm{M}_{\odot}\right)$ and the shock stalls into an accretion shock. Furthermore, in spherical symmetry (1D), it has been shown using Boltzmann neutrino transfer and the best physics that the delayed neutrino mechanism does not work either [23-25]. In 1D, the bounce shock stalls and is not revived, though an increase of only $\sim 25 \%$ in neutrino heating would lead to explosion! Such an increase could arise from as-yet-unknown neutrino effects or overturning motions in the inner core that could boost the neutrino luminosity. The former are unlikely and the latter have yet to be demonstrated.

However, in 2D, but using less sophisticated neutrino transfer (e.g., gray; 1D transport along multiple radial rays), numerous calculations result in explosions [8,9,26], although sometimes weak. These calculations demonstrate that neutrino-driven convection in the so-called "gain" region near the shock [27] increases the efficiency of neutrino energy deposition, increases the size of the gain region, and facilitates explosion.

Figure 1 depicts the various important regions. It is neutrino energy deposition in the gain region, not radiation pressure, that is important and if this deposition is adequate explosions are easy. The increase in efficiency in $2 \mathrm{D}$ can be traced to the increase in the average dwell time $\left(\tau_{a d v}\right)$ of the matter in the gain region before matter settles into the cooling region and onto the inner core. If the heating time $\left(\tau_{H}\right)$ is small compared with the hydrodynamic accretion/advection time into the interior $\left(\tau_{a d v}\right)$, then the object explodes [17]. If $\tau_{H}$ is larger than $\tau_{a d v}$, then the object does not explode. This qualitative condition is simple and multi-D effects increase $\tau_{a d v}$. In fact, recent 3D calculations, still using 1D transport along radial rays, reveal that $3 \mathrm{D}$ is marginally better than $2 \mathrm{D}$. However, even this $3 \mathrm{D}$ explosion is underenergetic by factors. We speculate $(\S 4)$ that a little rotation (a "rotation boost") can make the difference in explosion energy and viability, that rotation is the "missing factor," though how much and how it is or needs to be distributed has yet to be determined. Nevertheless, current calculations of the delayed neutrino mechanism have revealed that it is so close to working that we would be surprised if it weren't essentially correct.

Note that delay is a good thing, for it ensures that the remaining neutron star has sufficient mass to explain measured pulsar masses and that the ejected material is not too neutron-rich to be inconsistent with nucleosynthetic constraints. Recent calculations [17] demonstrate that using Boltzmann $\nu_{e}$ and $\bar{\nu}_{e}$ transport in the delayed explosion context raises the $\mathrm{Y}_{e}$ of ejected matter as it emerges to values equal to or slightly greater than 


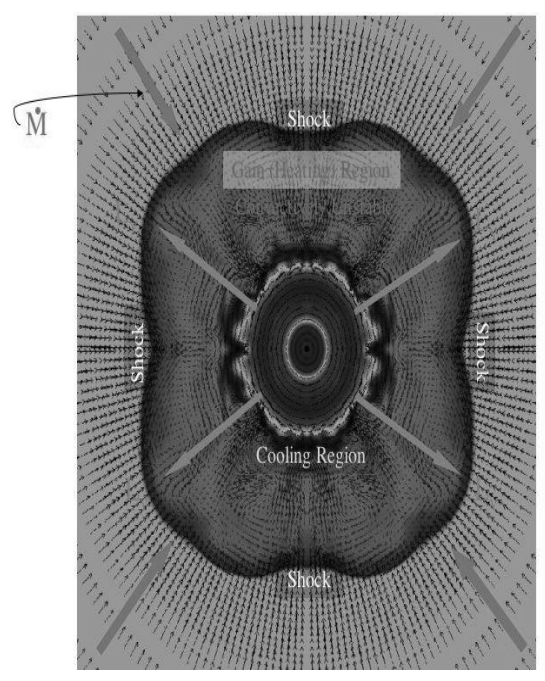

Figure 1. A schematic of the stalled shock wave after bounce, indicating the various important regions. They include the gain region, the cooling region, and the shocked region. Shown are the neutrino luminosity emerging from the core, the shock wave, and mass accretion. Note that the gain region is also the unstable region. See text for a discussion.

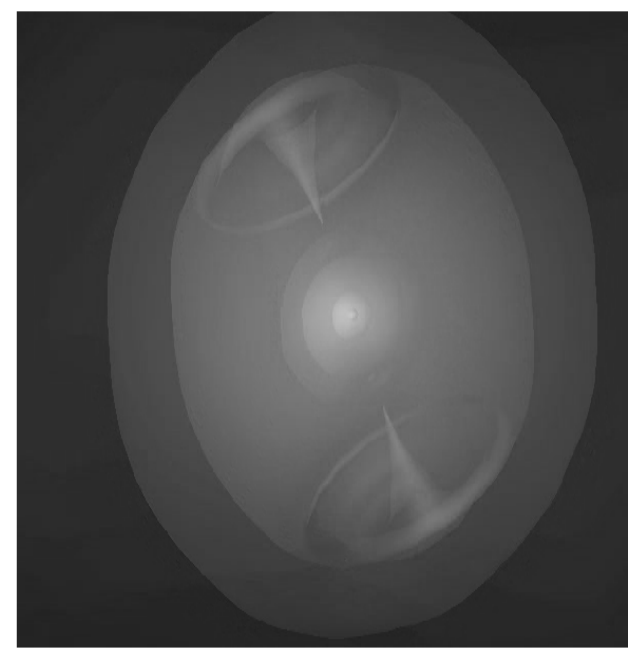

Figure 2. A snapshot of a rapidly rotating collapse and bounce simulation in $2 \mathrm{D}$, rendered in $3 \mathrm{D}$ with nested layers of isodensity contours from $10^{8} \mathrm{gm} \mathrm{cm}^{-3}$ to $10^{13} \mathrm{gm} \mathrm{cm}^{-3}$. The funnel along the poles due to the centrifugal barrier created after bounce by the rotation of the collapsing Chandrasekhar core is clearly seen. Taken from Burrows, Ott, \& Meakin (2003).

0.5. This does not happen with gray or more approximate schemes $[8,9]$, nor for rapid explosions with little delay. 


\subsection{A Digression on the Basics of Supernova Energetics}

The supernova explosion is a phenomenon of the outer mantle at ten times the radius of a cold neutron star. Though the binding energy of a cold neutron star is $\sim 3 \times 10^{53} \mathrm{ergs}$ and the supernova explosion energy is near $10^{51} \mathrm{ergs}$, a comparison of these two energies and the large ratio that results are not very relevant. More relevant are the binding energy of the mantle (interior to the shock or, perhaps, exterior to the neutrinospheres) and the neutrino energy radiated during the delayed phase. These are both at most a few $\times 10^{52}$ ergs, not $\sim 3 \times 10^{53} \mathrm{ergs}$, and the important ratio that illuminates the neutrino-driven supernova phenomenon is $\sim 10^{51}$ ergs divided by a few $\times 10^{52}$ ergs. This is $\sim 5-10 \%$, not the oft-quoted $1 \%$, a number which tends to overemphasize the sensitivity of the neutrino mechanism to neutrino and numerical details. Five to ten percent of the neutrino energy coursing through the semi-transparent region is required, not one percent. Importantly, the optical depth to neutrino absorption in the gain region is of order $\sim 0.1$. The product of the sum of the $\nu_{e}$ and $\bar{\nu}_{e}$ neutrino energy emissions in the first 100's of milliseconds and this optical depth gives a number near $10^{51}$ ergs. Perhaps, this is not a coincidence.

\section{Core Rotation: The Missing Factor?}

The amplification of the angular velocity due to large changes in the radius of a given mass shell during collapse implies that rotation may be a factor in core collapse and in the explosion mechanism. There are a few aspects of rotating collapse that distinguish it from spherical collapse: 1) Rotation provides centrifugal support and lowers the effective gravity in the core, increasing the radius of the stalled shock and the size of the gain region. Since ejection is inhibited by the deep potential well, this consequence of rotation alone might facilitate explosion. 2) Rotation enhances the neutrino flux and heating rate along the poles, relative to those at the equator [28] (§5), perhaps facilitating a bipolar explosion $[14,15,29]$. 3) Due to the centrifugal barrier, rotation results in an anisotropy in the mass accretion flux after bounce. If the rotation is "rapid," funnel structures are generated at the poles (see Figure 2; [15]). This, and the enhancement of neutrino energy deposition along the poles $(\S 5)$, suggest that the neutrino-driven mechanism could be along the poles. Clearly, bipolarity is not an exclusive signature of MHD driven explosions and may be a natural consequence of the neutrino-driven mechanism with rotation. However, if the rotation rate is too low for these polar effects to manifest themselves significantly, rotation might still lower the effective gravity and mass accretion rate in the equatorial regions enough to lead to an equatorial, not a polar, explosion. What obtains computational and physically has yet to be determined, but the potential variety rotation introduces should stimulate much research. Finally, 4) rotation generates vortices (Eddington-Sweet) that might dredge up heat from below the neutrinospheres and thereby enhance the driving neutrino luminosities.

Of course, the actual magnitude of all these effects is a function of the initial rotational profile. Nevertheless, the crucial questions that remain to be answered are: How much rotation is necessary to validate the basic paradigm we have outlined? What do we mean by "rapid," "modest," or slow rotation? At what critical rotation rate is the transition from neutrino-driven to "MHD"-driven? "Modest" rotation may not be as rapid as some think, but these questions are the subjects of current research. 
Even if the core is left with a high spin rate and we needed to shed angular momentum via viscous torques [17] to achieve the low inferred initial pulsar spin rates, it is not true that the "initial" kinetic energy of this shed core spin would necessarily find itself in the supernova. Viscous dissipation conserves angular momentum and total energy, but not kinetic energy. A differentially rotating object at a given angular momentum has free energy in its differential motion. The rotational energy could end up in heat [17], which in the protoneutron star and its inner envelope would be radiated in neutrinos. Therefore, a large fraction of the $10^{52}$ ergs of rotational energy in a 5-millisecond protoneutron star could be converted into neutrinos and would not have to power a super-energetic supernova. This would be a small fraction of the total radiated neutrino energy of a few $\times 10^{53}$ ergs. Angular momentum is still conserved.

\section{Rotation-Induced Anisotropy of the Neutrino Field and Heating Profile}

For our core-collapse studies, we have constructed the first 2D multi-group, multi-angle, time-dependent radiation/hydrodynamics code in astrophysics. In addition to being 6dimensional $(1$ (time) +2 (space) +2 (angles) +1 (energy groups $))$, VULCAN/2D [30] has an ALE (Arbitrary Lagrangian-Eulerian) structure with remap, is axially-symmetric, can handle rotation, is flux-conservative, smoothly matches to the diffusion limit, and is implicit in its Boltzmann solver, The implicit hydro variant has yet to be tested. It also has a 2D MGFLD (multi-group, flux-limited diffusion) version that is computationally much faster and allows us to more quickly explore parameter space. What the code does not yet have are the velocity-dependent terms in the transport equation, such as the Doppler shift and aberration, though it does have the advection term. It also does not have energy redistribution due to inelastic scattering, though this will be incorporated in explicit fashion [25] in a subsequent version.

Here, we present some stills from a preliminary 2D MGFLD calculation of the effect of rotation on the neutrino radiation field. Sixteen energy groups were used in these early test calculations. In 2D, we simulated collapse, bounce, neutrino shock breakout, and the neutrino-driven convection stages for a $15-\mathrm{M}_{\odot}$ progenitor [31]. Movies of these simulations, in particular detailing the evolution of the neutrino flux vectors for various energy groups, are available from the first author upon request.

The results are summarized in Figures 3, 4, and 5. Figure 3 is a color map at 9.6 milliseconds after bounce of the entropy distribution, with the flux vectors for electron neutrinos at $2.5 \mathrm{MeV}$ superposed. Note that the vectors are longer along the poles than at the equator, reflecting the anisotropy of the neutrino emission induced by rotation. Figure 4 shows the same quantities, but with the flux vectors for the $13.9 \mathrm{MeV} \nu_{e}$ energy group superposed. As is clear from a comparison of Figures 3 and 4, the fluxes and flux anisotropy are functions of neutrino group. Furthermore, and importantly, both figures demonstrate that the entropy due to neutrino heating is larger along the poles than the equator, qualitatively verifying one of the effects of rotation discussed in $\S 4$, and in Shimizu et al. [28], Madokoro, Shimizu, \& Motizuki [32], and Kotake, Yamada, \& Sato [29], that could aid explosion. Not shown is the anisotropy of the neutrino energy density contours that are oblate in the interior, but prolate outside near the shock wave. The rotationally-induced oblateness of the matter in the inner core can easily be seen in Figures 


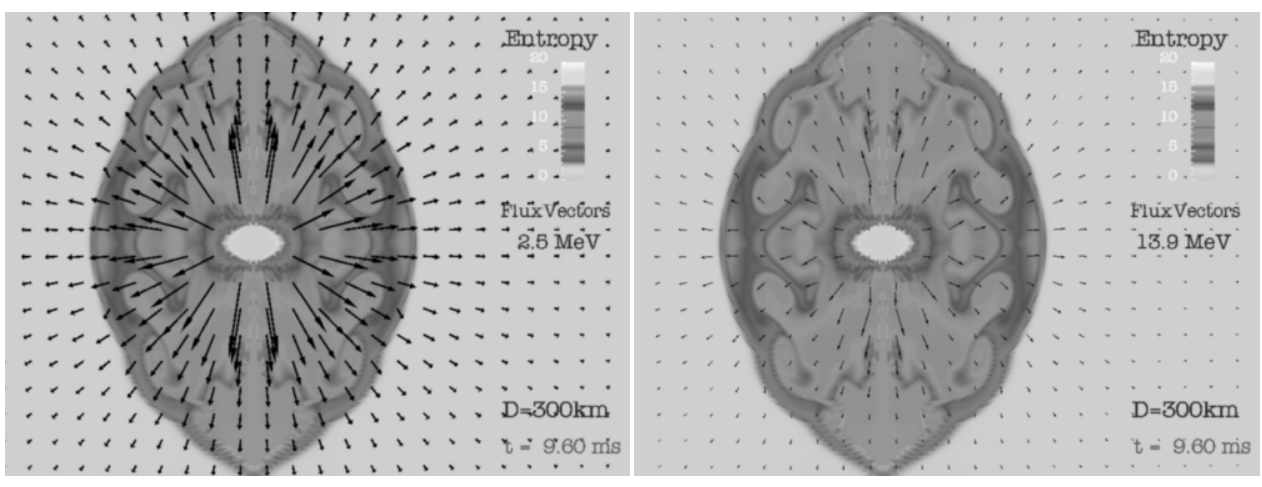

Figure 3. A snapshot at 9.6 milliseconds after bounce of a simulation of the core of a rotating $15-\mathrm{M}_{\odot}$ progenitor. The color map is of entropy and the vectors are flux vectors for the $2.5-\mathrm{MeV} \nu_{e} \mathrm{~s}$. See text for a discussion.
Figure 4. The same as Figure 3, but for the $13.9 \mathrm{MeV} \nu_{e}$ energy group.

3 and 4. This oblateness of the neutrino energy density in the interior is a consequence of von Zeipel's theorem concerning the coincidence of iso-effective-temperature surfaces and isopotential surfaces. Rotation makes the "photosphere" oblate. The prolateness of the neutrino energy density further out is related to the larger angle subtended by the core at the pole than the equator. These are the first consistent time-dependent calculations of this effect, though its basics have been investigated previously [33,34,28, 29,32]. Moreover, rotation has modified the convective plumes and created near bounce a barrel-shaped structure rotating on cylinders. Figure 5 shows a map of $\mathrm{Y}_{e}$ at 22.6 milliseconds after bounce, with the $7.8 \mathrm{MeV} \nu_{e}$ flux vectors superposed. At this early stage, for this calculation, the anisotropies of the $\mathrm{Y}_{e}$ and electron-capture-rate distributions are dramatic.

Soon, we hope to know the true effect of a given degree of rotation on the supernova itself. Whether the requisite rotation is provided by progenitor evolution has yet to be determined.

\section{Closing Remarks}

The theoretical study of supernova explosions is starting to couple multi-dimensional effects, neutrino radiation, rotational effects, B-fields, pulsar spins and kicks, GRBs, hypernovae, and observed supernova blast morphologies to obtain a consistent synthesis. That synthesis has not yet been accomplished, but its outlines are sharpening. In the next year, we hope to be able to determine the role of rotation and multi-dimensional transport effects on the supernova mechanism itself and answer many of the questions 


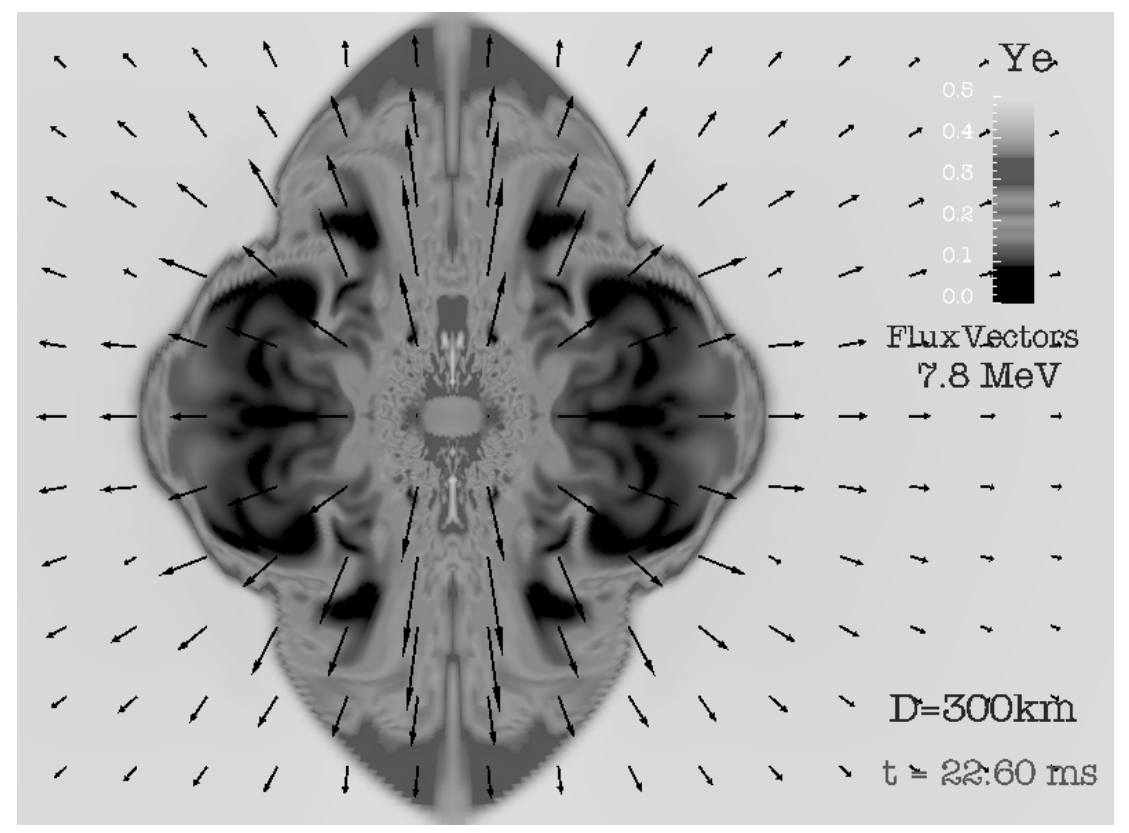

Figure 5. A color map of the $\mathrm{Y}_{e}$ distribution 22.6 milliseconds after bounce. Superposed are flux vectors for the $7.8 \mathrm{MeV} \nu_{e}$ energy group. See text for a discussion.

posed in this summary.

\section{REFERENCES}

1. P.A. Mazzali, et al., Ap. J. 599 (2003) L95.

2. P. Höflich, J.C. Wheeler, and L. Wang, Ap. J. 521 (1999) 179.

3. S. Akiyama, J.C. Wheeler, D. Meier, and I. Lichtenstadt, Ap. J. 584 (2003) 954.

4. K. Maeda, et al. Ap. J. 565 (2002) 405.

5. P. Podsiadlowski, P.A. Mazzali, K. Nomoto, D. Lazzati, and E. Cappellaro, Ap. J. 607 (2004) L17.

6. A.I. MacFadyen and S.E. Woosley, Ap. J. 524 (1999) 262.

7. L. Wang, D. Baade, P. Höflich, and J.C. Wheeler, Ap. J. 592 (2003) 457.

8. A. Burrows, J. Hayes, and B.A. Fryxell, Ap. J. 450 (1995) 830.

9. C.L. Fryer and M. Warren, Ap. J. 574 (2002) L65.

10. M. Rampp and H.-Th. Janka, Astr. Ap. 396 (2002) 331.

11. R. Buras, M. Rampp, H.-Th. Janka, and K. Kifonidis, Phys. Rev. Letts. 90 (2003) 241101.

12. L. Scheck, T. Plewa, H.-Th. Janka, K. Kifonidis, and E. Müller, Phys. Rev. Letts. 92 (2004) 011103. 
13. L. Wang, et al., Ap. J. 579 (2002) 671.

14. C.L. Fryer and A. Heger, Ap. J. 541 (2000) 1033.

15. A. Burrows, C.D. Ott, and C. Meakin, to be published in the proceedings of "3D Signatures in Stellar Explosions: A Workshop honoring J. Craig Wheeler's 60th birthday," held June 10-13, 2003, Austin, Texas, USA.

16. U. Hwang, et al., Ap. J. (2004), in press.

17. T.A. Thompson, E. Quataert, and A. Burrows, submitted to Ap. J. (2004), astro$\mathrm{ph} / 0403224$.

18. R. Willingale, J.A.M. Bleeker, K.J. van der Heyden, J.S. Kaastra, and J. Vink, Astr. Ap. 381 (2002) 1039.

19. R. Willingale, J.A.M. Bleeker, K.J. van der Heyden, and J.S. Kaastra, Astr. Ap. 398 (2003) 1021.

20. U. Hwang, A.E. Szymkowiak, R. Petre, and S. Holt, Ap. J. 560 (2001) L175.

21. J.R. Thorstensen, R.A. Fesen, and S. van den Bergh, A. J. 122 (2001) 297.

22. A. Burrows and J. Hayes, Phys. Rev. Letts. 76 (1996) 352.

23. A. Mezzacappa, M. Liebendörfer, O.E.B. Messer, W.R. Hix, F.-K. Thielemann, and S.W. Bruenn, Phys. Rev. Letts. 86 (2001) 1935.

24. M. Liebendörfer, A. Mezzacappa, F.-K. Thielemann, O.E.B. Messer, W.R. Hix, and S.W. Bruenn, Phys. Rev. D 63 (2001) 103004.

25. T.A. Thompson, A. Burrows, and P.A. Pinto, Ap. J. 592 (2003) 434.

26. M. Herant, W. Benz, W.R. Hix, C.L. Fryer, and S.A. Colgate, Ap. J. 435 (1994) 339.

27. H. Bethe and J.R. Wilson, Ap. J. 295 (1985) 14.

28. T. Shimizu, T. Ebisuzaki, K. Sato, and S. Yamada, Ap. J. 552 (2001) 756.

29. K. Kotake, S. Yamada, and K. Sato, Ap. J. 595 (2003) 304.

30. E. Livne, A. Burrows, R., Walder, T.A. Thompson, and I. Lichtenstadt, Ap. J. 609 (2004) 277.

31. S.E. Woosley and T.A. Weaver, Ap. J. Suppl. 101 (1995) 181.

32. H. Madokoro, T. Shimizu, and Y. Motizuki, (2004) astro-ph/0312624.

33. H.-Th. Janka and R. Mönchmeyer, Astr. Ap. 209 (1989) L5.

34. H.-Th. Janka and R. Mönchmeyer, Astr. Ap. 226 (1989) 69. 